\title{
ACTN3 genotype influences muscle performance through the regulation of calcineurin signaling
}

\author{
Jane T. Seto,,1,2 Kate G.R. Quinlan,,1,2 Monkol Lek,1,2 Xi Fiona Zheng,1,2 Fleur Garton,1,2 \\ Daniel G. MacArthur,1,2 Marshall W. Hogarth,1,2 Peter J. Houweling,1,2 Paul Gregorevic,3 \\ Nigel Turner, ${ }^{4,5}$ Gregory J. Cooney, ${ }^{4,5}$ Nan Yang,, ${ }^{1,2}$ and Kathryn N. North ${ }^{1,2,6}$

\begin{abstract}
'Institute for Neuroscience and Muscle Research, The Children's Hospital at Westmead, Sydney, Australia. ${ }^{2}$ Discipline of Paediatrics and Child Health, Faculty of Medicine, University of Sydney, Australia. ${ }^{3}$ Division of Metabolism and Obesity, Baker IDI Heart and Diabetes Institute, Victoria, Australia.

${ }^{4}$ Diabetes and Obesity Research Program, Garvan Institute of Medical Research, Darlinghurst, Australia. ${ }^{5}$ St. Vincent's Hospital Clinical School, University of New South Wales, Sydney, Australia. ${ }^{6}$ Murdoch Childrens Research Institute, The Royal Children's Hospital, Melbourne, Victoria, Australia.
\end{abstract}

\begin{abstract}
$\alpha$-Actinin-3 deficiency occurs in approximately $16 \%$ of the global population due to homozygosity for a common nonsense polymorphism in the ACTN3 gene. Loss of $\alpha$-actinin-3 is associated with reduced power and enhanced endurance capacity in elite athletes and nonathletes due to "slowing" of the metabolic and physiological properties of fast fibers. Here, we have shown that $\alpha$-actinin-3 deficiency results in increased calcineurin activity in mouse and human skeletal muscle and enhanced adaptive response to endurance training. $\alpha$-Actinin-2, which is differentially expressed in $\alpha$-actinin-3-deficient muscle, has higher binding affinity for calsarcin-2, a key inhibitor of calcineurin activation. We have further demonstrated that $\alpha$-actinin-2 competes with calcineurin for binding to calsarcin-2, resulting in enhanced calcineurin signaling and reprogramming of the metabolic phenotype of fast muscle fibers. Our data provide a mechanistic explanation for the effects of the ACTN3 genotype on skeletal muscle performance in elite athletes and on adaptation to changing physical demands in the general population. In addition, we have demonstrated that the sarcomeric $\alpha$-actinins play a role in the regulation of calcineurin signaling.
\end{abstract}

\section{Introduction}

$\alpha$-Actinin- 3 is one of the major components of the skeletal muscle Z-disk in fast-twitch muscle fibers (1) and interacts with multiple structural, metabolic, and signaling proteins $(2,3)$. Homozygosity for a common nonsense polymorphism in the ACTN3 gene (R577X) results in complete $\alpha$-actinin-3 deficiency in an estimated $16 \%$ of the global population (4) and is associated with variations in human muscle performance. The ACTN3 577XXnull genotype is markedly underrepresented in elite sprint and power athletes (5-9) and is associated with reduced muscle strength and sprint performance in nonathlete cohorts (10-13), suggesting that $\alpha$-actinin-3 deficiency has a detrimental effect on the optimal function of fast muscle fibers. In contrast, the ACTN3 $577 \mathrm{XX}$ genotype is overrepresented in elite endurance athlete cohorts $(5,14,15)$, suggesting a beneficial effect on endurance capacity. Recent studies in athletes and nonathletes further suggest that the ACTN3 genotype influences the adaptive response of skeletal muscle to exercise training $(10,16)$.

The Actn3 KO mouse model mimics the phenotypic effects of $\alpha$-actinin-3 deficiency in humans (17). The closely related sarcomeric isoform $\alpha$-actinin- 2 compensates for the absence of $\alpha$-actinin-3 and is expressed in all fiber types in Actn $3 \mathrm{KO}$ mice, similarly to ACTN3 577XX humans. Compared with WT mice, Actn3 KO mice have substantially lower grip strength, increased recovery from fatigue, and enhanced endurance exercise performance associated with increased levels of glycogen and a shift in fast muscle fiber properties toward a slow-twitch, oxidative phenotype, without

Conflict of interest: The authors have declared that no conflict of interest exists. Citation for this article: J Clin Invest. 2013;123(10):4255-4263. doi:10.1172/JCI67691. changes to fiber typing as defined by myosin heavy chains (17-19). The metabolic phenotype in the Actn $3 \mathrm{KO}$ mouse is similar to that seen in skeletal muscle following exercise training, suggesting that Actn $3 \mathrm{KO}$ muscle is "pretrained" for endurance performance.

The molecular basis for the effects of $\alpha$-actinin- 3 deficiency on fast muscle fiber properties is not yet known. One potential downstream target is the calcium- and calmodulin-dependent protein phosphatase calcineurin, which indirectly associates with sarcomeric $\alpha$-actinins at the $Z$-disk via their mutual binding to calsarcins (20-23). It is well established that calcineurin signaling plays a critical role in the remodeling of skeletal muscle; it specifically induces activation of the slow myogenic program and a shift in muscle fiber attributes toward a slow-twitch, oxidative phenotype $(24,25)$ and confers fatigue resistance in endurance running $(26)$. The expression of activated calcineurin in fast glycolytic skeletal muscles has been shown to induce a shift in glucose metabolism toward decreased glucose oxidation and increased insulin-stimulated glucose incorporation into glycogen (27). It has recently been shown that calsarcin-2, which, like $\alpha$-actinin-3, is expressed exclusively in fast-twitch muscle, inhibits calcineurin signaling in vivo. Mice deficient in calsarcin-2 display enhanced endurance capacity and their muscles exhibit a shift toward slower fiber properties, similar to the phenotype observed in the Actn3 KO mouse model (28). There were no changes in the expression of $\alpha$-actinin- 2 and -3 in the muscles of calsarcin- $2 \mathrm{KO}$ mice, raising the possibility that the $\alpha$-actinins act upstream of calsarcin- 2 as key regulators of muscle fiber phenotype (28). We hypothesized that the phenotypic changes observed in $\alpha$-actinin-3-deficient fast fibers are due to increased calcineurin activity, which in turn is regulated by the differential binding of calsarcin- 2 to sarcomeric $\alpha$-actinins. 
A
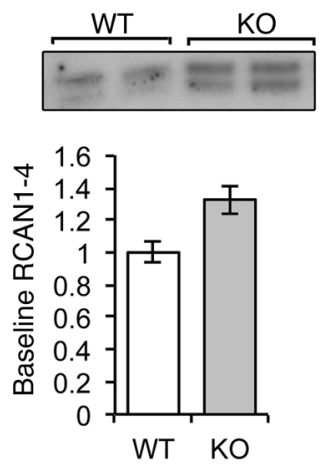

B
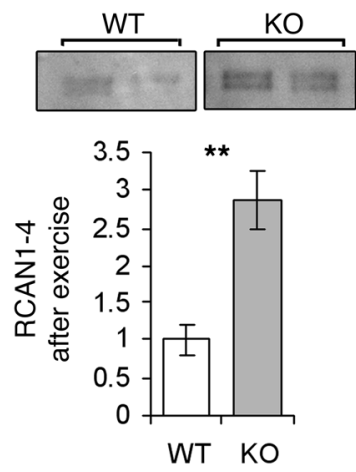

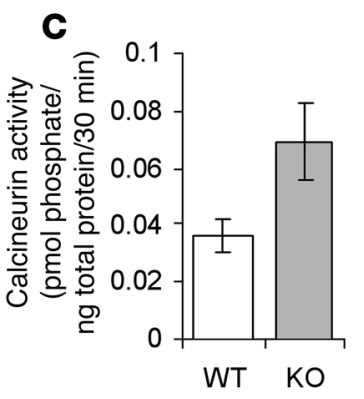

D

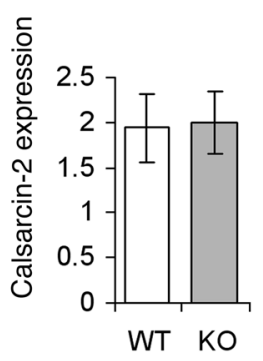

\section{Figure 1}

Calcineurin signaling is increased in $\alpha$-actinin-3-deficient muscles. (A) Muscles of KO mice on a C57BL/6J background showed a 1.3-fold increase in RCAN1-4 protein expression compared with WT muscles, suggesting increased calcineurin activity ( $P=0.057 ; n=3-4$ for both groups). (B) In exercised mice on a 129 background, RCAN1-4 was 2.9-fold upregulated in the KO group ( $P=0.004 ; n=6$ for both groups). (C) Direct assays of calcineurin activity confirmed a 1.9-fold increase in calcineurin signaling in exercised $\mathrm{KO}$ muscles $(P=0.093$; $n=6$ for both groups). (D) Calsarcin-2 expression was similar between WT and $\mathrm{KO}$ muscles (mean $\pm \mathrm{SEM}$; ${ }^{* *} P<0.01$, Mann-Whitney $U$ test). In $\mathbf{B}$ and $\mathbf{D}$, the lanes were run on the same gel but were noncontiguous.
In this study, we demonstrate that calcineurin activity is increased in the absence of $\alpha$-actinin- 3 and provide a molecular mechanism to explain the slower metabolic, physiological, and functional phenotypes associated with $\alpha$-actinin-3 deficiency. Increased calcineurin activity is also associated with increased muscle plasticity, as demonstrated by an enhanced adaptive response to endurance training in Actn3 $\mathrm{KO}$ mice and an increased switch in muscle fiber type from fast-twitch glycolytic fibers toward fast-twitch oxidative fibers. We further demonstrate that calsarcin-2, a key inhibitor of calcineurin activation, preferentially binds to $\alpha$-actinin- 2 over $\alpha$-actinin- 3 and that $\alpha$-actinin- 2 outcompetes calcineurin for binding to calsarcin-2. We propose that the upregulation of $\alpha$-actinin- 2 , in the absence of $\alpha$-actinin-3, releases calcineurin from calsarcin- 2 inhibition and alters the metabolic phenotype of fast glycolytic (2B) muscle fibers. Taken together, our data suggest a novel role for the sarcomeric $\alpha$-actinins in the phenotypic regulation of skeletal muscle fibers at baseline and in response to physical demands via modulation of calcineurin-dependent signaling.

\section{Results}

Calcineurin activity is increased in $\alpha$-actinin-3-deficient muscles. To determine whether the phenotypic changes in $\alpha$-actinin-3-deficient muscle are associated with increased calcineurin activity, we first examined the expression of RCAN1-4, a downstream regulator of calcineurin, which is specifically increased upon activation of calcineurin and, in turn, stimulates calcineurin signaling when expressed at physiological levels (29). Above physiological levels, RCAN1-4 also acts in a negative feedback loop to inhibit further calcineurin activity $(30,31)$. The expression of RCAN1 is tightly controlled by a cluster of 15 NFAT binding sites and is thus a sensitive endogenous calcineurin reporter gene (30). At baseline, Actn3 KO muscles showed a 1.3-fold increase in the levels of RCAN1-4 compared with WT muscles, however, this failed to reach formal significance $(P=0.057)$ (Figure $1 \mathrm{~A})$. As calcineurin is known to play a critical role in promoting the transition of fast-twitch muscle fibers toward a slow-twitch phenotype in response to low-amplitude, sustained calcium activity (32), we further examined the levels of RCAN1-4 in muscles from WT and KO mice 3 days after a single bout of endurance running; this approach has previously been used to identify gene expression changes in muscle in response to endurance exercise (33). Our results show that the muscles of Actn3 KO mice have significantly elevated expression of RCAN1-4 after exercise compared with the muscles of WT mice (2.9-fold; $P=0.004$ ) (Figure $1 \mathrm{~B}$ ), which is consistent with potentiated calcineurin activity in the absence of actinin-3.

To confirm that calcineurin activity is increased in unexercised and exercised Actn $3 \mathrm{KO}$ muscles, we directly assayed the total phosphatase activity and the calcium-independent phosphatase activity in lysates of muscles. Calcineurin activity is calcium dependent and is determined by subtracting the level of calcium-independent phosphatase activity from the total phosphatase activity. Consistent with RCAN1-4 results, exercised Actn3 KO muscles demonstrated a 1.9-fold increase in calcineurin activity compared with strain-matched WT muscles (Figure 1C). There were no differences in the activity of calcium-independent phosphatases between WT and Actn3 KO samples (data not shown), indicating a specific influence of sarcomeric $\alpha$-actinin isoform expression upon calcium-dependent phosphatase (calcineurin) activity in skeletal muscle.

Since calsarcin-2 has previously been demonstrated to inhibit calcineurin activity (28), we investigated whether differences in calcineurin activity between WT and Actn $3 \mathrm{KO}$ muscles were associated with altered expression of the calsarcin-2 protein. We found that calsarcin-2 expression was not altered in Actn3 KO muscles compared with WT muscles (Figure 1D), suggesting that sarcomeric $\alpha$-actinins regulate calcineurin activity via a mechanism that does not depend on differential expression of calsarcin-2.

$\alpha$-Actinin-3 deficiency potentiates the adaptive response to endurance training. Increased calcineurin activity is associated with activation of the slow myogenic program and an enhanced response of skel- 


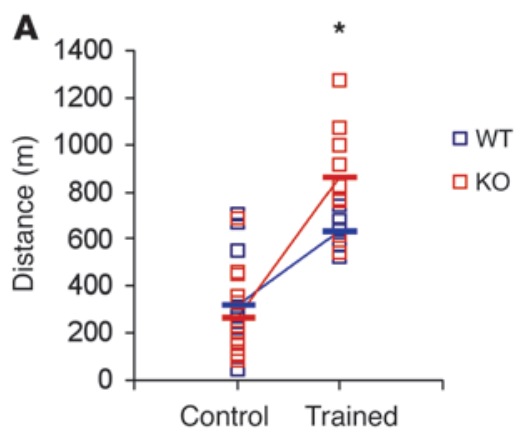

Figure 2

Endurance performance and grip strength of WT and KO mice after endurance training. (A) The endurance performance of untrained control WT (cWT) and control KO mice (cKO) were not significantly different at 15 to 16 weeks of age, however, the endurance performance of KO mice was significantly greater than WT mice after training. Trained WT mice (tWT) ran twice the distance of the cWT mice, while trained $\mathrm{KO}$ mice (tKO) ran 3.3 times the distance of untrained $\mathrm{KO}$ mice. (B) No significant increase in grip strength was detected in either WT or KO mice with endurance training. WT mice demonstrated greater grip strength than $\mathrm{KO}$ mice in both the untrained and trained groups (mean \pm SEM; * $P<0.05$ for WT versus tKO; ${ }^{* \star} P<0.01$, Mann-Whitney $U$ test; $n=6-8$ for all groups).

etal muscle to increased physical demands. Since $\alpha$-actinin-3 deficiency is associated with enhanced endurance performance in elite athletes $(5,14)$, we hypothesized that increased calcineurin activity in $\alpha$-actinin-3-deficient muscles would contribute to a greater adaptive response to endurance training. To test this hypothesis, we compared the performance of WT and Actn $3 \mathrm{KO}$ mice following 4 weeks of treadmill training with age-matched (16 weeks of age), untrained control cohorts (Figure 2A). We have previously shown that KO mice run farther than WT mice at 8 weeks of age (17), but this difference in baseline endurance performance is lost by 16 weeks of age (34). However, after 4 weeks of exercise training, 16-week-old Actn3 KO mice were able to run significantly farther than the trained WT mice $(P=0.026)$. Specifically, trained WT mice ran twice the distance of WT controls, while trained Actn3 KO mice ran 3.3 times farther than Actn3 KO controls. Significant interaction between genotype and training confirms an increased response to endurance training in Actn $3 \mathrm{KO}$ mice $(F=5.32(1,38)$, $P=0.027)$, consistent with a significantly increased response to training in Actn3 KO mice. In contrast, grip strength was not significantly altered with training in either WT or Actn3 KO mice (Figure 2B). Similar to our previous results, untrained WT mice had a greater average grip strength compared with untrained Actn3 KO mice $(P=0.003)(18)$. A similar trend was observed for the trained group $(P=0.088)$.

Actn3 KO muscles exhibit increased oxidative enzyme activity and altered fiber type adaptation in response to endurance training. To examine the response to endurance training in greater detail, we assessed the activity of metabolic enzymes in the quadriceps muscles of sedentary and trained WT and Actn $3 \mathrm{KO}$ mice. The quadriceps muscle was examined because it is one of the most activated muscles during uphill running (35). Consistent with previously published data, untrained control Actn3 KO mice showed higher activities of enzymes involved in the glycolytic pathway (hexokinase [HK]) (Figure 3A), the mitochondrial aerobic pathway (citrate synthase $[\mathrm{CS}]$, succinate dehydrogenase $[\mathrm{SDH}]$ ) (Figure 3, B and $\mathrm{C}$ ), and the fatty acid oxidation pathway ( $\beta$-hydroxyacyl CoA dehydrogenase
[BHAD]) (Figure 3D) (18). Following endurance training, the activity of CS, SDH, BHAD, and $\mathrm{HK}$ increased in both WT and Actn3 KO muscles. Western blot analysis of the mitochondrial protein cytochrome c oxidase subunit IV (COX IV) similarly showed an increase in expression in response to endurance training (Figure 3E). The activities of oxidative enzymes in WT muscle after training were similar to the muscles of sedentary Actn3 KO mice, supporting our proposal that Actn $3 \mathrm{KO}$ muscle is "pretrained" for endurance performance.

Since increased calcineurin activity promotes adaptation toward the slow myogenic program in response to physical demands, we investigated whether Actn3 KO muscles demonstrate greater changes in fiber size and fiber type proportions in response to endurance training in comparison with WT muscles. Consistent with previously published data (18), fiber type proportions (as defined by myosin heavy chain isoforms) did not differ between the muscles of untrained WT and Actn3 KO mice (Supplemental Figure 1; supplemental material available online with this article; doi:10.1172/ JCI67691DS1). However, the effect of endurance training on fiber type proportions differed between the WT and Actn $3 \mathrm{KO}$ muscles. Endurance training had a minimal effect on the proportions of $2 \mathrm{~B}$ and $2 \mathrm{X}$ fibers in WT quadriceps; changes did not reach formal statistical significance (2B: $-6.5 \%, P=0.082 ; 2 \mathrm{X}: 2.0 \%, P=0.537)$. In the Actn3 KO quadriceps, there was a $9.1 \%$ decrease in $2 \mathrm{~B}$ fibers $(P=0.004)$ and a $5.2 \%$ increase in $2 \mathrm{X}$ fibers $(P=0.04)$. Similar increases in $2 \mathrm{~A}$ fibers (in which $\alpha$-actinin-3 is not usually expressed) were observed in both WT and KO muscles (4.8\% WT, $P=0.008$; 3.8\% KO, $P=0.015$ ). These results suggest that Actn3 KO muscles have an increased propensity for fiber-type conversion toward a slower phenotype. Training also induced greater hypertrophy of $2 \mathrm{X}$ and $2 \mathrm{~A}$ fibers in Actn3 KO muscles (2X: 24.4\%, $P=0.051$; 2A: 26.5\%, $P=0.051)$ compared with WT (2X: $13.5 \%, P=0.093$; 2 : $5.5 \%, P=0.589)$, such that $2 \mathrm{X}$ fibers were found to be significantly larger in $\mathrm{KO}$ muscles compared with WT muscles after training (Supplemental Figure 2).

To examine the combined effect of changes in fiber size and number in each fiber type, we analyzed the total cross-sectional area (CSA) occupied by fibers of each type as a proportion of the overall muscle cross section (Figure $3 \mathrm{~F}$ ). Interestingly, control Actn $3 \mathrm{KO}$ muscles showed a trend toward lower 2B CSA proportions $(-3.3 \%, P=0.093)$ and significantly higher $2 \mathrm{X}$ CSA proportion $(2.5 \%, P=0.041)$ compared with WT muscles. Endurance training did not significantly alter the CSA proportions of any fiber type in WT muscles. In contrast, Actn $3 \mathrm{KO}$ muscles showed a significantly reduced 2B CSA of $8.0 \%(P=0.041)$ and an increased 2X CSA proportion of $5.5 \%(P=0.009)$ in response to training, resulting in further differences between WT and Actn3 KO in 2B (WT: $85.1 \%, A c t n 3 \mathrm{KO}: 77.6 \% ; P=0.004$ ) and $2 \mathrm{X}$ (WT: $6.3 \%$, Actn3 KO: $13.2 \% ; P=0.004)$ CSA proportions. This was confirmed by significant genotype and training interaction for $2 \mathrm{X}$ CSA using twoway ANOVA $(F=5.71(1,19), P=0.027)$. Together, these results suggest that Actn3 KO muscles demonstrate greater plasticity in relation to fiber-type shifts in response to endurance training.

Muscles from $\alpha$-actinin-3-deficient humans exhibit increased calcineurin activity. To confirm that our observations in Actn3 $\mathrm{KO}$ mouse muscle are relevant to and reproducible in humans, we examined muscle biopsies from humans with ACTN3 577RR 

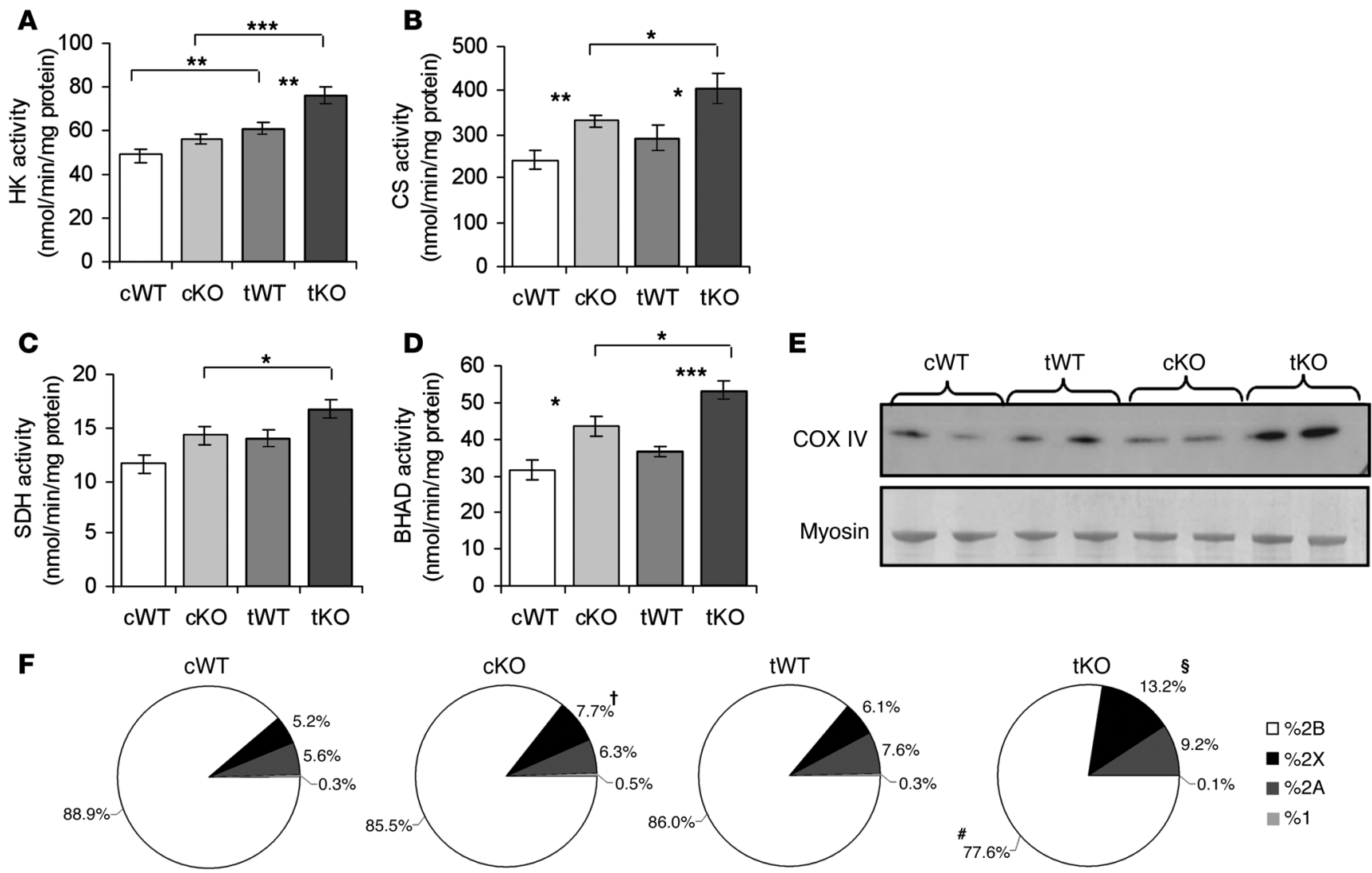

Figure 3

Enhanced response to exercise training in KO mice. Training increased activities of (A) HK, (B) CS, (C) SDH, and (D) BHAD in both WT and KO muscles. (E) Exercise training increased the level of COX IV in both WT and KO muscles, with KO muscles demonstrating higher levels of COX IV than WT in both untrained and trained states. (F) The CSA occupied by each fiber type is presented as a proportion of the overall muscle cross section. CKO muscles demonstrated 3.3\% lower 2B CSA proportions $(P=0.093)$ and $2.5 \%$ higher $2 \mathrm{X}(P=0.041)$ CSA proportions compared with cWT muscles. tWT muscles showed no significant alterations in CSA proportions for any fiber type compared with cWT muscles, while tKO muscles demonstrated significant decreases in 2B and increases in 2X CSA compared with cKO muscles (mean \pm SEM; ${ }^{*} P<0.05$; ${ }^{* *} P<0.01$; ${ }^{* \star \star} P<0.001 ;{ }^{\dagger} P<0.05$ for cWT versus cKO; ${ }^{P} P<0.05 ; \AA P<0.01$ for $\mathrm{cKO}$ versus tKO, Mann-Whitney $U$ test; $n=6-7$ muscles; greater than 5,000 fibers per muscle for all groups).

genotypes (WT, $n=5$, all female, age range 27-67 years, mean age 47 years) and $577 \mathrm{XX}(\alpha$-actinin-3 deficient, $n=6$, all female, age range 33-77 years, mean age 49 years) for differences in calcineurin activity. Consistent with our findings in the Actn $3 \mathrm{KO}$ mouse, muscles from ACTN3 577XX humans showed increased RCAN1-4 expression compared with the muscles of ACTN3 577RR humans $(P=0.004)$ (Figure 4).

Calsarcin-2 preferentially binds to $\alpha$-actinin-2. The expression of calsarcin-2 (which inhibits calcineurin activity; ref. 28) did not differ between the muscles of WT and Actn3 KO mice and hence cannot account for the altered calcineurin activity associated with $\alpha$-actinin-3 deficiency. Since the sarcomeric $\alpha$-actinins and calcineurin share the same $\mathrm{N}$-terminal binding site on calsarcin (23), we hypothesized that the observed differences in calcineurin activity between WT and Actn $3 \mathrm{KO}$ muscles could be associated with a differential binding affinity between calsarcin- 2 and the $\alpha$-actinin isoforms. Since previous studies of the interaction of calsarcin- 2 with the sarcomeric $\alpha$-actinins have not differentiated between $\alpha$-actinin-2 and -3 (22), we sought to define the binding affinity of the calsarcins to $\alpha$-actinin- 2 and -3 individually using a yeast two-hybrid assay.
Our results showed that the $\mathrm{N}$-terminal region of calsarcin-2 (amino acids $1-110$ and $1-90$ ) binds to both $\alpha$-actinin- 2 and $\alpha$-actinin-3, but there is preferential binding for $\alpha$-actinin-2 (Figure $5 \mathrm{~A}$ ). We also examined the binding affinities of the sarcomeric $\alpha$-actinins for the corresponding regions of calsarcin-1 and calsarcin-3, but did not observe growth above background levels (empty vector; pGAD10), suggesting that interaction with the sarcomeric $\alpha$-actinins in this region is unique to calsarcin- 2 . However, fulllength calsarcin-1 and calsarcin-3, as well as calsarcin-2, interact with the rod domain of full-length $\alpha$-actinin- 2 and $\alpha$-actinin-3. There was no preferential binding to $\alpha$-actinin- 2 or $\alpha$-actinin- 3 for the calsarcins at this site (Supplemental Figure 3). These results suggest that while the rod domain of sarcomeric $\alpha$-actinins interacts readily with the $\mathrm{C}$ terminus of the calsarcins, the $\mathrm{N}$-terminal of calsarcin- 2 shows a preference for binding with $\alpha$-actinin-2.

We further quantified the interactions between the $\mathrm{N}$-terminal of calsarcin-2 (1 to 110) to full-length $\alpha$-actinin- 2 and $\alpha$-actinin-3 in the yeast two-hybrid system by liquid $\beta$-galactosidase assays (Figure 5B). The $\beta$-galactosidase reaction for both $\alpha$-actinin-2 and $\alpha$-actinin- 3 was greater than that of the empty vector alone, indicating that calsarcin- 2 binds to both sarcomeric $\alpha$-actinins. 
$\begin{array}{lllllllllllllllll}\text { RR } & \text { RR } & \text { RR } & \text { RR } & \text { RR } & X X & X X & X X & X X & X X & X X\end{array}$

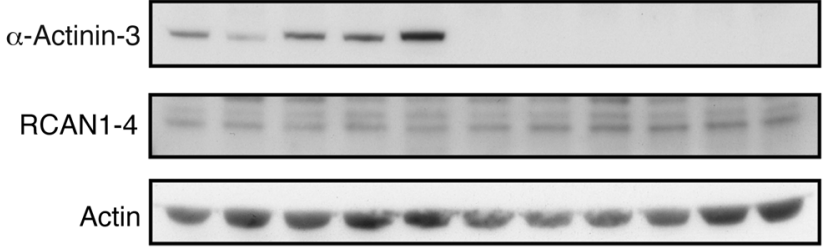

Figure 4

Calcineurin signaling is increased in $\alpha$-actinin-3-deficient human muscles. Muscles of ACTN3 $577 X X$ humans showed a significant increase in the expression of RCAN1-4 when compared with ACTN3 577RR humans ( $n=5$ and 6 females, respectively; $P=0.004$; mean $\pm \mathrm{SEM}$; ${ }^{* *} P<0.01$, Mann-Whitney $U$ test). Histogram shows the relative density of bands normalized to actin and with the RR mean set to 1 (AU). 577RR, homozygous for the ACTN3 R allele.

However, the interaction with full-length $\alpha$-actinin-2 was 13.6-fold higher compared with $\alpha$-actinin-3, consistent with a stronger affinity for binding of calsarcin-2 to full-length $\alpha$-actinin- 2 in preference to $\alpha$-actinin-3.

$\alpha$-Actinin- 2 competes with calcineurin for binding to calsarcin-2. Since $\alpha$-actinin-2 is the only isoform expressed in Actn $3 \mathrm{KO}$ mouse muscle and human ACTN3 577XX muscle and is upregulated more than 2-fold compared with WT mouse muscle (36), we hypothesized that the preferential binding of calsarcin- 2 to $\alpha$-actinin- 2 could influence the tethering and inhibition of calcineurin by calsarcin-2. To test this hypothesis, we cotransfected FLAG-tagged cal-

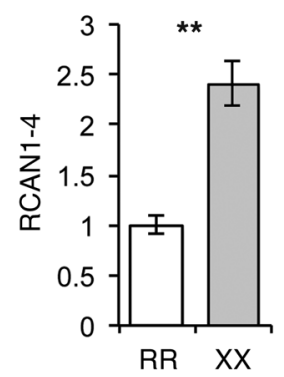

sarcin-2 and Myc-tagged calcineurin into COS-1 cells in the presence of increasing amounts of $\alpha$-actinin-2 (Figure 5, $\mathrm{C}$ and D, and Supplemental Figure 4); cell extracts were subsequently immunoprecipitated with anti-FLAG affinity gel to extract proteins that had bound to calsarcin-2. As predicted, our results show that the amount of calcineurin that coimmunoprecipitates with calsarcin- 2 is inversely proportional to the level of $\alpha$-actinin- 2 present, suggesting that increasing concentrations of $\alpha$-actinin- 2 inhibit binding of calcineurin to calsarcin-2. This, in turn, would result in increased calcineurin activity.

RCAN1-4 is increased in WT mouse muscle overexpressing $\alpha$-actinin- 2 . To confirm that differential expression of $\alpha$-actinin- 2 directly results in enhanced calcineurin signaling in vivo, we overexpressed $\alpha$-actinin- 2 by injecting the tibialis anterior muscles of WT mice with recombinant AAV6 vectors packaging a CMV-hACTN2 or a CMV-hACTN3 expression cassette. Western blot analyses showed that $\alpha$-actinin-2 was increased by 3.1-fold in muscles treated with CMV-hACTN2 compared with control WT muscles injected with empty vector (Figure 6A). Consistent with our in vitro data, RCAN1-4 was also increased (2.7-fold) compared with control WT muscles, indicating that increased calcineurin signaling is associated with increased $\alpha$-actinin-2 expression (Figure 6B). In contrast, RCAN1-4
A

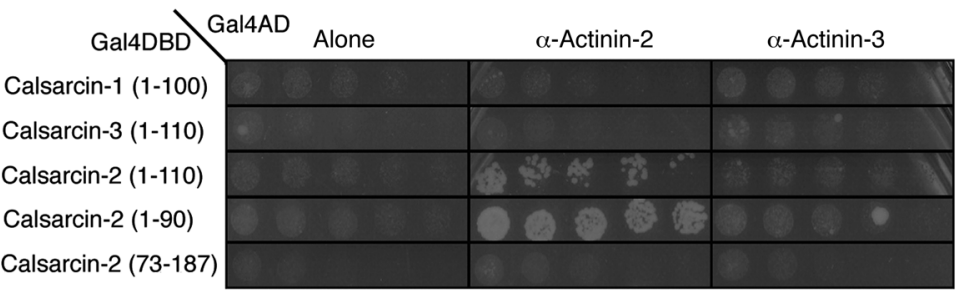

C

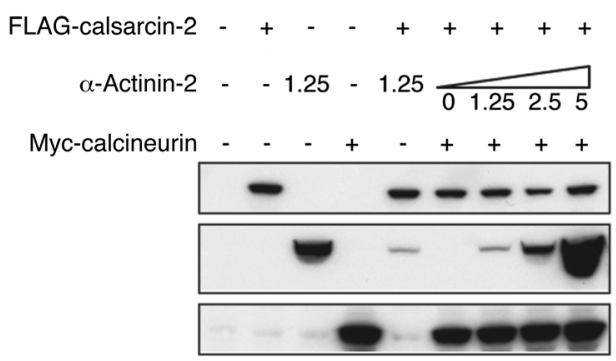

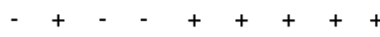
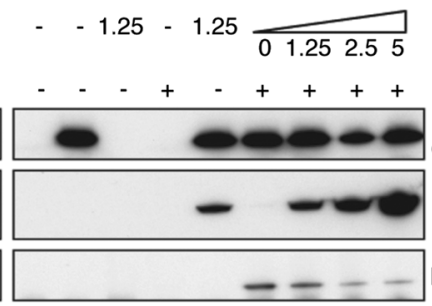

B

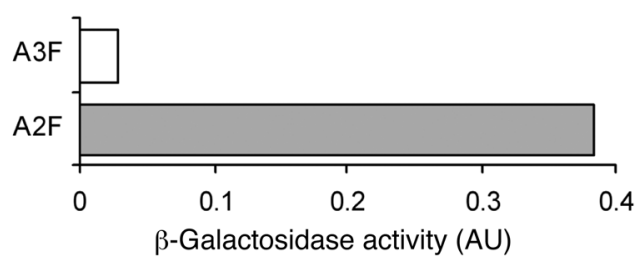

$\beta$-Galactosidase activity (AU)

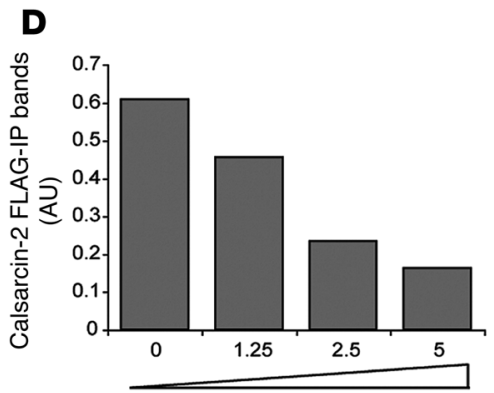

$\alpha$-Actinin-2 construct transfected $(\mu \mathrm{g})$

\section{Figure 5}

$\alpha$-Actinin-2 expression influences the inhibition of calcineurin activity via preferential binding to calsarcin-2. (A) The N-terminal calsarcin-2 constructs, but not the corresponding $\mathrm{N}$-terminal region on calsarcin-1 and -3 , interact with $\alpha$-actinin-2 in yeast two-hybrid assays. In addition, there was higher-affinity binding of calsarcin-2 to $\alpha$-actinin-2 than to $\alpha$-actinin-3; this was a 13.6 -fold difference when quantitated by liquid $\beta$-galactosidase assays (B). (C) COS-1 cells were transfected with or without vectors encoding FLAG-calsarcin-2 and Myc-calcineurin and varying amounts $(0-5 \mu \mathrm{g})$ of untagged $\alpha$-actinin-2. Whole-cell extracts (5\% input) showed the expression of each protein in COS-1 cells. Immunoprecipitations using anti-FLAG M2 affinity gel demonstrated decreasing coimmunoprecipitation of calcineurin with calsarcin-2, with increasing concentrations of $\alpha$-actinin-2, indicative of $\alpha$-actinin-2 competitive inhibition of the calsarcin-2 interaction with calcineurin. (D) Densitometric quantitation of the decrease in calcineurin coimmunoprecipitation by calsarcin-2 with increasing amounts of $\alpha$-actinin-2 from the final four lanes for the FLAG immunoprecipitation blots (presented as a ratio of calcineurin/calsarcin-2). 
A
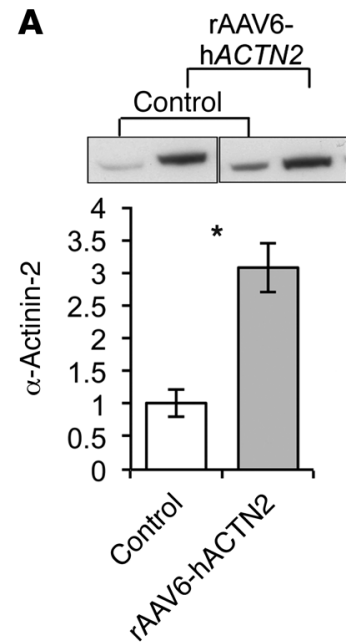

B
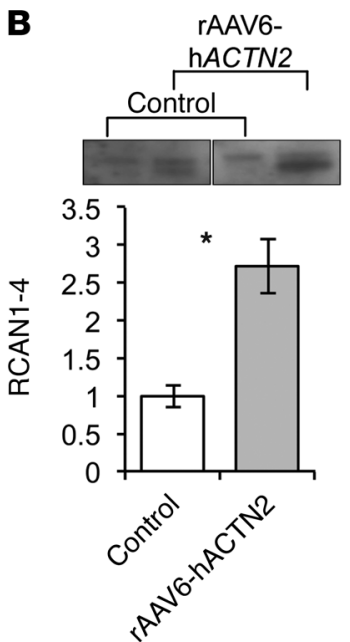

C

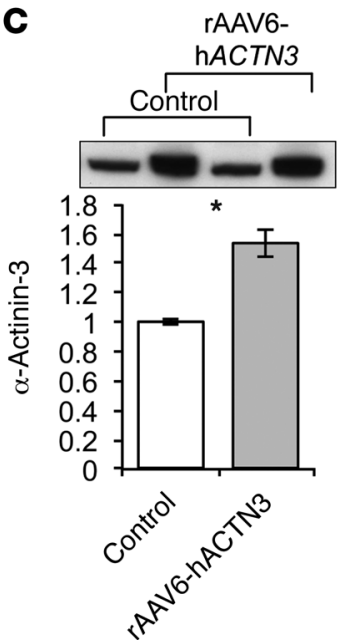

D

hACTN3
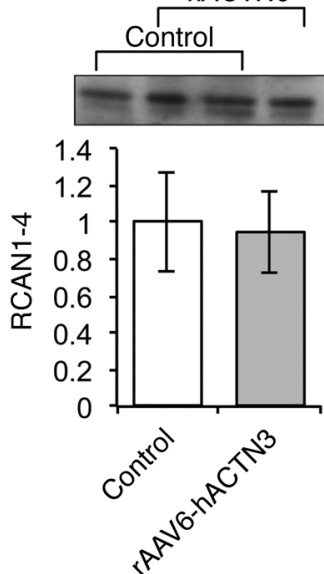

Figure 6

Overexpression of $\alpha$-actinin-2 in mouse muscle increases RCAN1-4 expression. (A and B) Overexpression of $\alpha$-actinin-2 (3.1-fold) through i.m. injection of $1 \times 10^{11}$ vector genomes $(\mathrm{vg})$ of rAAV6-CMV-mActn2 resulted in a 2.7-fold increase in RCAN1-4 expression $(P=0.028)$. (C and D) In contrast, a 1.5-fold overexpression of $\alpha$-actinin-3 through injection of $1 \times 10^{11} \mathrm{vg}$ of rAAV6-CMV-mActn3 did not significantly alter RCAN1-4 expression in injected muscles (mean $\pm \mathrm{SEM} ;{ }^{*} P<0.05$, Mann-Whitney $U$ test; $n=4$ for all samples). In $\mathbf{A}$ and $\mathbf{B}$, the lanes were run on the same gel but were noncontiguous.

expression was not significantly altered in muscles injected with CMV-hACTN3, despite demonstrating a 1.5-fold upregulation of $\alpha$-actinin-3 (Figure 6, C and D).

\section{Discussion}

Our finding that $\alpha$-actinin-2 competes with calcineurin for binding with calsarcin- 2 indicates that the $\alpha$-actinins act cooperatively with calsarcin-2 in skeletal muscle to regulate calcineurin signaling. Since $\alpha$-actinin- 2 is the only sarcomeric $\alpha$-actinin isoform expressed in the Actn3-null muscles of KO mice and ACTN3 577XX humans, we propose that increased calcineurin signaling in $\alpha$-actinin-3-deficient muscles is the result of the liberation of calcineurin from the inhibitory influence of calsarcin-2 (summarized in Figure 7). This could account for the observed increase in calcineurin activity in Actn 3 $\mathrm{KO}$ muscles and, in turn, for the shift toward increased oxidative metabolism in fast muscle fibers and enhanced endurance capacity at baseline, as well as an enhanced adaptive response and greater shift in fiber type following endurance training. Furthermore, our studies suggest a novel and important role for the $\alpha$-actinins in the phenotypic regulation of muscle fibers and provide tantalizing insights into the specialized functions of $\alpha$-actinin- 2 and $\alpha$-actinin-3. The sarcomeric $\alpha$-actinins diverged 250-300 million years ago following gene duplication, and ACTN3 has since developed restricted expression in fast muscle fibers (37). While the sarcomeric $\alpha$-actinins have been traditionally classified as "structural" muscle proteins, it is increasingly evident that they also have significant roles in the regulation of metabolic and signaling pathways. The relative amounts of $\alpha$-actinin- 2 and $\alpha$-actinin-3 in slow and fast muscle fibers likely influence the downstream characteristics of individual fibers in terms of their metabolic profile, contractile properties, and fiber-type specification.

\section{Methods}

Animals. Mice used were of a 129X1/SvJ or C57BL/6J genetic background. The metabolic and performance phenotypes that were previously reported in $129 \mathrm{X} 1 / \mathrm{SvJ}(17,18)$ have been replicated and confirmed in the C57BL/6J and C57BL/10ScSn backgrounds that were bred for ten generations (Supplemental Figure 5). All animals were fed food and water ad libitum and were maintained on a 12-hour light/12-hour dark cycle from 7:00 am to 7:00 pm.

Immunoblotting. Muscle sections were cut from frozen muscles, and BCA protein assays were performed on homogenates to allow equal sample loading for protein content. Samples were loaded onto precast minigels (Invitrogen) and separated by SDS-PAGE, then transferred to polyvinylidene fluoride membranes (Millipore). These were then blocked with 5\% skim milk, $1 \times$ PBS, $0.1 \%$ Tween 20 , probed with the indicated antibodies, and developed with ECL reagents (Amersham Biosciences). The primary antibodies used include: RCAN/DSCR1 (1:500; D6694; Sigma-Aldrich); calsarcin-2 (1:10,000; C22920; BD Biosciences); COX IV (1:2,000; 20E8; Molecular Probes); porin (1:5,000; 20B12; Molecular Probes); $\alpha$-actinin-2 (1:500,000; 4A3; gift from A. Beggs, Children's Hospital Boston, Boston Massachusetts, USA); and $\alpha$-actinin-3 (1:1,500; 5A2; gift from A. Beggs, Children's Hospital Boston). Following probing, PVDF membranes were stained with Coomassie brilliant blue (Sigma-Aldrich) to generate final myosin loading controls. Densitometry of immunoblot bands was performed using ImageJ image processing software (NIH).

Calcineurin assay. A calcineurin assay kit (207007; Calbiochem) was used according to the manufacturer's instructions for a 30-minute reaction time on muscle homogenates. Results were normalized to total protein, as determined by BCA assay.

Endurance performance testing and training. Endurance performance of 6 WT and $9 \mathrm{KO}$ male mice were tested by treadmill running using a previously described protocol (17) on a motorized treadmill (Columbus Instruments). Mice were tested at 10 weeks and 16 weeks of age, 3 days after the conclusion of the training period. All animals were trained and tested by an operator blinded to the mouse genotype. Mice were tested and trained between 9:00 am and 12:00 pm within the first 6 hours of their dark cycle. Endurance training by motorized treadmill running began when mice were 12 weeks of age, 5 days per week for 4 weeks, with training intensity initiated at an $8 \%$ grade at 15 meters per minute for the first 2 days and increased to a $10 \%$ grade thereafter. The running speed was increased to 20 meters per minute by day 8 of training, and the duration of training 


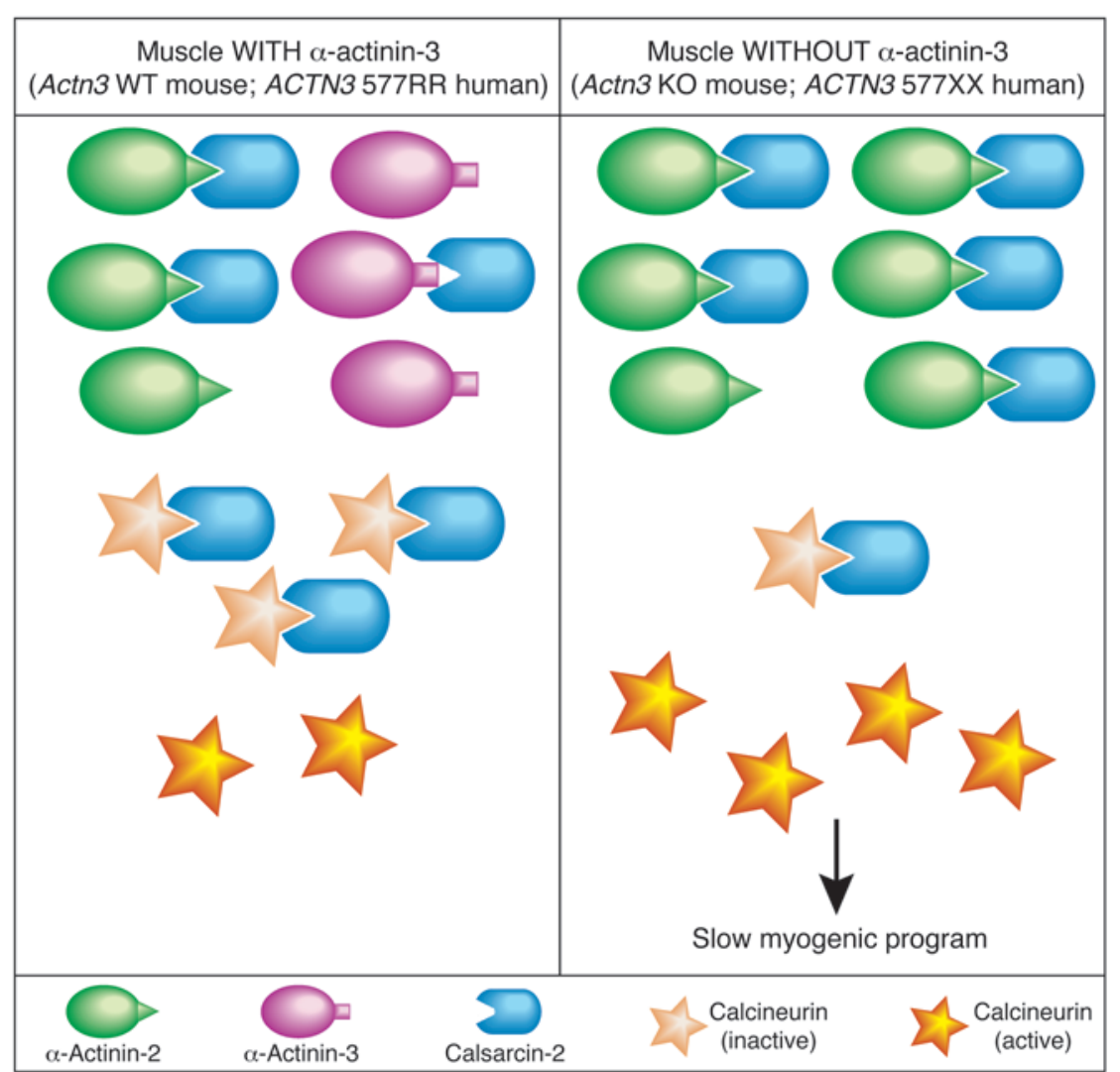

\section{Figure 7}

Schematic of sarcomeric $\alpha$-actinin regulation of calcineurin signaling. $\alpha$-Actinin- 2 is differentially expressed in muscles without $\alpha$-actinin-3. Higher-affinity binding of calsarcin-2 for $\alpha$-actinin-2 over $\alpha$-actinin- 3 and competitive binding of $\alpha$-actinin-2 for calsarcin-2 lead to the increased release of calcineurin from calsarcin-2 inhibition in the absence of $\alpha$-actinin-3. We propose that increased free (active) calcineurin activates downstream signaling and the slow myogenic program in $\alpha$-actinin-3-deficient muscle. was increased from 45 to 75 minutes by day 11 , such that mice would run 1.5 kilometers per day during the final 2 weeks of training. Mice were closely monitored for the duration of the training and gently nudged when needed to encourage continued participation in the training to completion. To ensure the health of the mice, the body weight of each mouse was monitored at the beginning of each week, and each mouse was inspected for injuries prior to and after each round of treadmill running.

Grip strength tests. The forearm grip strength of mice was tested using a grip strength meter (Columbus Instruments) according to a previously described method (18).

Enzyme assays. The activity of lactate dehydrogenase (LDH) (Enzyme Commission [EC] number 1.1.1.27), BHAD (EC 1.1.1.35), CS (EC 4.1.3.7), SDH (EC 1.3.5.1), and HK (EC 2.7.1.1) in WT and KO muscles was determined using previously described methods (18). For all assays, the linearity with time and dependence on the amount of extract added were confirmed. All assays for a particular enzyme were performed in triplicate on the same day. Activities for each sample in the absence of substrate were measured for each sample and were subtracted from these values.

Fiber measurements and fiber typing. Fiber measurements and fiber typing were performed on immunostained muscle sections using MetaMorph software (Molecular Devices), as previously described (38). For immunohistochemistry analysis, transverse $8-\mu \mathrm{m}$ sections were cut from the midsection of frozen quadriceps muscles. All sections were blocked with AffiniPure Fab Fragment Goat Anti-mouse IgG (1:25; Jackson ImmunoResearch) for 1 hour to prevent cross-reaction with endogenous mouse antibodies. Sections were then incubated for 1 hour at room temperature with primary antibodies against myosin heavy chain $2 \mathrm{~B}$ (used neat, BF-F3; DSHB, University of Iowa, Iowa City, Iowa, USA), 2A (used neat, SC71; DSHB, University of Iowa), and type 1 (1:300; MAB1628; Chemicon), which were prelabeled using the Zenon labeling kit (Molecular Probes) according to the manufacturer's instructions. All samples were also costained with dystrophin (dys6-10; 1:2,000; L. Kunkel, Boston Children's Hospital, Harvard Medical School, Boston Massachusetts, USA) to define fiber borders. Images were captured using an Olympus BX50 microscope and ProgRes software (SciTech).

cDNA cloning and sequencing. All PCR inserts for yeast two-hybrid analysis were amplified from the human skeletal muscle cDNA and subcloned into pGBT9 and pGAD10 vectors (Clontech). Primer design for calsarcin-1 (NM_016599), calsarcin-2 (NM_021245), calsarcin-3 (NM_133371), $\alpha$-actinin-2 (NM_001103), and $\alpha$-actinin-3 (NM_001104) were based on their respective GenBank template sequences indicated here in parentheses. Sequences of the completed constructs were confirmed by DNA sequencing.

Yeast two-hybrid analysis. Yeast two-hybrid analysis was performed as indicated in the Yeast Protocols Handbook (Clontech). The pGAD10 $\alpha$-actinin-2 (aa 1-894) and $\alpha$-actinin-3 (aa 1-901) constructs were cotransformed with various pGBT9 constructs containing calsarcin-1 (aa 1-100), calsarcin-2 (aa 1-110), or calsarcin-3 (aa 1-110) inserts. Transformants were plated and incubated for 3 days at $30^{\circ} \mathrm{C}$ on minimum yeast medium lacking leucine and tryptophan. Transformants were then spotted as a dilution series on minimum yeast medium lacking leucine, tryptophan, and histidine and were incubated at $30^{\circ} \mathrm{C}$. The plates were observed over 5 days to assess the growth strength.

Liquid $\beta$-galactosidase assay. The liquid $\beta$-galactosidase assay was carried out according to the method outlined in the Yeast Protocols Handbook (Clontech). Transformants were grown in $5 \mathrm{ml}$ of selective liquid media overnight at $30^{\circ} \mathrm{C}$. Two milliliters of culture was transferred to $8 \mathrm{ml}$ of yeast extract/peptone/dextrose (YPD) medium and incubated for an additional 5 hours. Culture ( $1.5 \mathrm{ml}$ of each) was spun, and the pellet was washed and then resuspended in $100 \mu \mathrm{l}$ of buffer $1(23.8 \mathrm{mg} / \mathrm{ml} \mathrm{HEPES}$, $9 \mathrm{mg} / \mathrm{ml} \mathrm{NaCl}, 0.65 \mathrm{mg} / \mathrm{ml} \mathrm{L}$-aspartate, $10 \mathrm{mg} / \mathrm{ml} \mathrm{BSA}, 0.05 \%$ Tween $20, \mathrm{v} / \mathrm{v}$, 
$\mathrm{pH}$ 7.25). Cells were lysed in five rounds of freeze-thawing in liquid nitrogen followed by the addition of $700 \mu \mathrm{l}$ of buffer 1 containing chlorophenol red $\beta$-D-galactopyranoside $(1.36 \mathrm{mg} / \mathrm{ml})$, and the mixture was incubated at $30^{\circ} \mathrm{C}$ until the samples turned red. The reaction was stopped by the addition of $500 \mu \mathrm{l}$ of $3 \mathrm{mM} \mathrm{ZnCl}_{2}$, and absorbance was read at $570 \mathrm{~nm}$. For each sample, AUs were calculated to correct for culture $\mathrm{OD}$ and incubation time.

Competitive coimmunoprecipitation. COS-1 cells were maintained in DMEM supplemented with $10 \%$ FCS. Confluent cells in a $10-\mathrm{cm}$ tissue culture dish were transiently transfected with the following amount of expression plasmids: $0.5 \mu \mathrm{g}$ of pMT3-FLAG-calsarcin-2, $0.25 \mu \mathrm{g}$ of pMT3-Myccalcineurin subunit $\mathrm{A}$, and an increasing dosage $(0,1.25,2.5$, and $5 \mu \mathrm{g})$ of pMT3- $\alpha$-actinin-2, using the FuGENE 6 transfection reagent (Roche Molecular Biochemicals). Empty pMT3 vector was added to appropriate transfections to ensure that the total micrograms of plasmid transfected was the same for all wells. This resulted in the expression of calsarcin-2 recombinant protein fused with an N-terminal FLAG epitope and calcineurin subunit A fused with an $\mathrm{N}$-terminal Myc epitope. Forty-eight hours after transfection, cells were harvested in $250 \mu \mathrm{l}$ of NP-40 lysis buffer containing $50 \mathrm{mM}$ Tris ( $\mathrm{pH} 8.0$ ), $150 \mathrm{mM} \mathrm{NaCl}, 1 \% \mathrm{NP}-40,0.2 \mathrm{mM}$ PMSF, and protease inhibitor (Sigma-Aldrich). Cells were incubated in NP-40 lysis buffer at $4^{\circ} \mathrm{C}$ for 30 minutes, and cell debris was removed by centrifugation. Ten microliters of this whole-cell extract was reserved as $5 \%$ protein input. The remaining $200 \mu \mathrm{l}$ of whole-cell extracts was precleared by incubating with Protein G Sepharose beads (GE Healthcare). The unbound portion of the whole-cell extract was immunoprecipitated for 1 hour at $4^{\circ} \mathrm{C}$ using Anti-FLAG M2 Affinity Gel (Sigma-Aldrich). Beads were washed with NP-40 lysis buffer prior to protein elution with SDS-DTT. The eluent was separated on SDS-PAGE and immunoblotted using antiFLAG (Sigma-Aldrich), anti-Myc (Santa Cruz Biotechnology Inc.), or 4B3 rabbit antibody (gift from A. Beggs from Children's Hospital Boston) for calsarcin-2, calcineurin subunit $A$, and $\alpha$-actinin-2, respectively.

Generation of rAAV6-CMV-hACTN2 and rAAV6-CMV-hACTN3 expression cassettes. Full-length human ACTN2 and ACTN3 were cloned into recombinant AAV6 expression cassettes containing AAV2 inverted terminal repeats using standard cloning techniques. The coding sequences of $\alpha$-actinin-2 and $\alpha$-actinin- 3 were cloned into the SacII site of the PAAV-CMV-SV40 plasmid. Methodologies for control vector creation, HEK293 cell transfection for rAAV6 vectors, vector purification, and titer quantification can be found in Gregorevic et al. (39).

Intramuscular delivery of $\mathrm{rAAV} 6$ expression cassettes. To transduce skeletal muscles in vivo, recombinant adenoassociated viral vectors (rAAV6 vectors) were injected directly into the tibialis anterior of WT mice using Hamilton syringes. Mice were anesthetized with isoflurane, and $1 \times 10^{11}$ vector genomes of either rAAV-aa2 or rAAV-aa3 were injected in $30 \mu \mathrm{l}$ of HBSS directly into the anterior compartment of the hind limb. The contralateral limb (used as a control) was injected with the empty vector lacking a functional gene. Four weeks after injection, mice were sacrificed via cervical dislocation, and the tibialis anterior muscles were excised and processed.

Statistics. Statistics were performed using SPSS software (version 15; SPSS Inc.). Because of small sample sizes, normality could not be assumed, so nonparametric statistics were applied. Kruskal-Wallis tests were applied for multiple group comparisons, followed by post-hoc Mann-Whitney $U$ tests for pairwise group comparisons. Two-way ANOVA was also applied to determine whether genotype influences the training response. All results are presented as the mean \pm SEM. The $\alpha$ level was set at $P<0.05$.

Study approval. This study was approved by the Animal Care and Ethics Committee of the Children's Medical Research Institute and The Children's Hospital at Westmead and by the Sydney Children's Hospitals Network Human Research Ethics Committee. Informed consent was obtained from all individuals included in this research.

\section{Acknowledgments}

This project was funded in part by a grant from the National Health and Medical Research Council of Australia (NHMRC) (1002033). J.T. Seto and K.G.R. Quinlan are supported by Early Career Fellowships from the NHMRC of Australia (1036656 and 0511981). M. Lek and F. Garton are supported by Australian Postgraduate Awards. X.F. Zheng is supported by a Dora Lush Biomedical Scholarship from the NHMRC of Australia (1038991). N. Turner is supported by a Future Fellowship from the Australian Research Council. G.J. Cooney is supported by a Research Fellowship from the NHMRC of Australia. P. Gregorevic is supported by a Senior Research Fellowship sponsored by Pfizer Australia. The Baker IDI Heart and Diabetes Institute is supported in part by the Operational Infrastructure Support Program of the Victorian Government. We thank Hongwei Qian for the manufacture of the rAAV vectors.

Received for publication November 7, 2012, and accepted in revised form July 19, 2013.

Address correspondence to: Kathryn N. North, Murdoch Childrens Research Institute, The Royal Children's Hospital, Flemington Road, Parkville, Victoria 3052, Australia. Phone: 613.8341.6226; Fax: 613.9348.1391; E-mail: kathryn.north@mcri.edu.au.

Jane T. Seto's present address is: Department of Neurology, University of Washington School of Medicine, Seattle, Washington, USA.

Monkol Lek's and Daniel G. MacArthur's present address is: Analytic and Translational Genetics Unit, Massachusetts General Hospital, Boston, Massachusetts, USA.
1. MacArthur DG, North KN. A gene for speed? The evolution and function of $\alpha$-actinin-3. Bioessays. 2004;26(7):786-795.

2. Houweling PJ, North KN. Sarcomeric $\alpha$-actinins and their role in human muscle disease. Future Neurol. 2009;4(6):731-743.

3. Lek M, North KN. Are biological sensors modulated by their structural scaffolds? The role of the structural muscle proteins alpha-actinin-2 and alpha-actinin-3 as modulators of biological sensors. FEBS Lett. 2010;584(14):2974-2980.

4. North KN, Yang N, Wattanasirichaigoon D, Mills $\mathrm{M}$, Easteal S, Beggs AH. A common nonsense mutation results in alpha-actinin-3 deficiency in the general population. Nat Genet. 1999;21(4):353-354.

5. Yang N, et al. ACTN3 genotype is associated with human elite athletic performance. Am J Hum Genet.
2003;73(3):627-631.

6. Niemi AK, Majamaa K. Mitochondrial DNA and ACTN3 genotypes in Finnish elite endurance and sprint athletes. EurJ Hum Genet. 2005;13(8):965-969.

7. Roth SM, Walsh S, Liu D, Metter EJ, Ferrucci L, Hurley BF. The ACTN3 R577X nonsense allele is under-represented in elite-level strength athletes. Eur J Hum Genet. 2008;16(3):391-394.

8. Druzhevskaya AM, Ahmetov II, Astratenkova IV, Rogozkin VA. Association of the ACTN3 R577X polymorphism with power athlete status in Russians. Eur J Appl Physiol. 2008;103(6):631-634.

9. Papadimitriou ID, Papadopoulos C, Kouvatsi A Triantaphyllidis C. The ACTN3 gene in elite Greek track and field athletes. Int J Sports Med. 2008; 29(4):352-355.

10. Clarkson PM, et al. ACTN3 genotype is associated with increases in muscle strength in response to resistance training in women. J Appl Physiol. 2005; 99(1):154-163.

11. Moran $\mathrm{CN}$, et al. Association analysis of the ACTN3 R577X polymorphism and complex quantitative body composition and performance phenotypes in adolescent Greeks. Eur J Hum Genet. 2007; 15(1):88-93.

12. Walsh S, Liu D, Metter EJ, Ferrucci L, Roth SM. ACTN3 genotype is associated with muscle phenotypes in women across the adult age span. J Appl Physiol. 2008;105(5):1486-1491.

13. Vincent B, et al. ACTN3 (R577X) genotype is associated with fiber type distribution. Physiol Genomics. 2007;32(1):58-63.

14. Eynon N, et al. ACTN3 R577X polymorphism and Israeli top-level athletes. Int J Sports Med. 2009; 
30(9):695-698.

15. Eynon N, et al. The ACTN3 R577X polymorphism across three groups of elite male European athletes. PLoS ONE. 2012;7(8):e43132.

16. Pimenta EM, et al. The ACTN3 genotype in soccer players in response to acute eccentric training. Eur J Appl Physiol. 2012;112(4):1495-1503.

17. MacArthur DG, et al. Loss of ACTN3 gene function alters mouse muscle metabolism and shows evidence of positive selection in humans. Nat Genet. 2007;39(10):1261-1265.

18. MacArthur DG, et al. An Actn3 knockout mouse provides mechanistic insights into the association between $\alpha$-actinin-3 deficiency and human athletic performance. Hum Mol Genet. 2008; 17(8):1076-1086.

19. Quinlan KG, et al. $\alpha$-Actinin-3 deficiency results in reduced glycogen phosphorylase activity and altered calcium handling in skeletal muscle. Hum Mol Genet. 2010;19(7):1335-1346.

20. Faulkner G, et al. FATZ, a filamin-, actinin-, and telethonin-binding protein of the Z-disc of skeletal muscle. J Biol Chem. 2000;275(52):41234-41242.

21. Takada F, et al. Myozenin: an alpha-actinin- and gamma-filamin-binding protein of skeletal muscle $\mathrm{Z}$ lines. Proc Natl Acad Sci U S A. 2001;98(4):1595-1600.

22. Frey N, Richardson JA, Olson EN. Calsarcins, a novel family of sarcomeric calcineurin-binding proteins. Proc Natl Acad Sci U S A. 2000;97(26):14632-14637.

23. Frey N, Olson EN. Calsarcin-3, a novel skeletal muscle-specific member of the calsarcin family, inter- acts with multiple Z-disc proteins. J Biol Chem. 2002; 277(16):13998-14004.

24. Chin ER, et al. A calcineurin-dependent transcriptional pathway controls skeletal muscle fiber type. Genes Dev. 1998;12(16):2499-2509.

25. Delling U, Tureckova J, Lim HW, De Windt LJ, Rotwein P, Molkentin JD. A calcineurin-NFATc3-dependent pathway regulates skeletal muscle differentiation and slow myosin heavy-chain expression. Mol Cell Biol. 2000;20(17):6600-6611.

26. Jiang LQ, Garcia-Roves PM, de Castro Barbosa T, Zierath JR. Constitutively active calcineurin in skeletal muscle increases endurance performance and mitochondrial respiratory capacity. Am J Physiol Endocrinol Metab. 2010;298(1):E8-E16.

27. Long YC, Glund S, Garcia-Roves PM, Zierath JR. Calcineurin regulates skeletal muscle metabolism via coordinated changes in gene expression. J Biol Chem. 2007;282(3):1607-1614.

28. Frey N, et al. Calsarcin-2 deficiency increases exercise capacity in mice through calcineurin/NFAT activation. J Clin Invest. 2008;118(11):3598-3608.

29. Kingsbury TJ, Cunningham KW. A conserved family of calcineurin regulators. Genes Dev. 2000; 14(13):1595-1604.

30. Yang J, et al. Independent signals control expression of the calcineurin inhibitory proteins MCIP1 and MCIP2 in striated muscles. Circ Res. 2000; 87(12):E61-E68.

31. Rothermel BA, Vega RB, Williams RS. The role of modulatory calcineurin-interacting proteins in calcineurin signaling. Trends Cardiovasc Med. 2003;13(1):15-21.

32. Dolmetsch RE, Lewis RS, Goodnow CC, Healy JI. Differential activation of transcription factors induced by $\mathrm{Ca} 2+$ response amplitude and duration. Nature. 1997;386(6627):855-858.

33. Mahoney DJ, Parise G, Melov S, Safdar A, Tarnopolsky MA. Analysis of global mRNA expression in human skeletal muscle during recovery from endurance exercise. FASEB J. 2005;19(11):1498-1500.

34. Seto JT, et al. The effect of $\alpha$-actinin-3 deficiency on muscle aging. Exp Gerontol. 2011;46(4):292-302.

35. Sloniger MA, Cureton KJ, Prior BM, Evans EM. Lower extremity muscle activation during horizontal and uphill running. J Appl Physiol. 1997; 83(6):2073-2079.

36. Seto JT, et al. Deficiency of alpha-actinin-3 is associated with increased susceptibility to contraction-induced damage and skeletal muscle remodeling. Hum Mol Genet. 2011;20(15):2914-2927.

37. Lek M, Quinlan KG, North KN. The evolution of skeletal muscle performance: gene duplication and divergence of human sarcomeric alpha-actinins. Bioessays. 2010;32(1):17-25.

38. Garton F, Seto JT, North KN, Yang N. Validation of an automated computational method for skeletal muscle fibre morphometry analysis. Neuromuscul Disord. 2010;20(8):540-547.

39. Gregorevic P, et al. Systemic delivery of genes to striated muscles using adeno-associated viral vectors. Nat Med. 2004;10(8):828-834. 